\title{
Relationship between Extracellular Polysaccharide and Benzene Tolerance of Rhodococcus sp. 33
}

\author{
Tomoko Aizawa1, Brett A. Neilan², Iain Couperwhite' ${ }^{2}$, Makoto Urai ${ }^{1}$, Hirosi Anzai ${ }^{3}$, \\ Noriyuki Iwabuchi ${ }^{1}$, Mutsuyasu Nakajima ${ }^{1 *}$ and Michio Sunairi ${ }^{1}$ \\ ${ }^{1}$ Laboratory of Molecular Microbiology, Department of Applied Biological Science, College of Bioresource Sciences, Nihon \\ University, 1866 Kameino, Fujisawa, Kanagawa 252-8510, Japan \\ ${ }^{2}$ School of Biotechnology and Biomolecular Sciences, University of New South Wales, Sydney, N.S.W. 2052, Australia \\ ${ }^{3}$ Laboratory of Applied Biochemistry, Department of Agriculture, Junior College, Nihon University, 1866 Kameino, Fujisawa, \\ Kanagawa 252-8510, Japan
}

(Received Jan. 20, 2005 / Accepted Feb. 16, 2005)

\begin{abstract}
Benzene is one of the most toxic and prevailing environmental pollutants. Rhodococcus sp. 33 can tolerate and efficiently degrade various concentrations of benzene. Under either resting or growth conditions, rough mutant strains derived from strain 33 were more sensitive to benzene than the mucoidal parent strain. The rough strains did not produce extracellular polysaccharides (EPS), whereas the parental strain 33 did so in large quantity (33 EPS). By the addition of 33 EPS to the rough strains, both the survival and growth of the rough strains in media containing benzene were improved. The 33 EPS was found to be an acidic polysaccharide containing D-galactose, D-glucose, D-mannose, D-glucuronic acid, and pyruvic acid at a molar ratio of 1: 1: 1: 1: 1. These data suggest that the 33 EPS plays an important role in the benzene tolerance in Rhodococcus sp. 33, especially helping the cells to survive an initial challenge with benzene.
\end{abstract}

\section{INTRODUCTION}

Rhodococcus sp. 33 was isolated from a contaminated site near a chemical plant at Port Botany in Sydney, Australia, based on its ability to tolerate and efficiently degrade various concentrations of benzene up to its maximal solubility $(1.8 \mathrm{~g} / \mathrm{l})$ in aqueous media ${ }^{1)}$. To elucidate the mechanism by which strain 33 tolerates such high concentrations of benzene, Gutierrez et al. examined the effects of benzene on cellular fatty acid composition of strain $33^{2)}$, because benzene is widely accepted to cause an increase in fluidity of cell membranes ${ }^{3)}$ and a number of reports have shown that various hydrocarbon-tolerant microorganisms produce qualitative and quantitative changes in their membrane fatty acids in response to toxic organic compounds to decrease the fluidity of their cell membranes $^{3-5)}$. The results showed an increase in the ratio of saturated/unsaturated cellular fatty acids in the presence of benzene from 1.3 (for mannitol-grown cells) to 1.5 (for benzene-grown cells). As expected from the increase in the ratio of saturated/unsaturated cellular fatty acids, the fluidity of the membranes of strain 33 decreased after 18-20 $\mathrm{h}$ incubation in the presence of benzene, as found by measuring the generalized polarization of the fluorescent membrane probe laurdan by fluorescence spectroscopy ${ }^{6}$. These data suggested a decrease in the fluidity of the membranes caused by changes in the cellular fatty acid composition as a possible mechanism for benzene tolerance.
Although the fluidity of the membranes was observed to decrease after the cells had been exposed to benzene for approximately $18-20 \mathrm{~h}$, the immediate effect of benzene on the membranes was an increase in fluidity, which was observed for more than $6 \mathrm{~h}$. These data suggest that an adaptive response, set to mitigate benzene toxicity, is induced only after long exposure and that with respect to the organism's tolerance during the first $6 \mathrm{~h}$, Rhodococcus sp. 33 may have a well-adapted inherent mechanism to survive an initial challenge with benzene.

Recently, Iwabuchi et al. reported that a mucoidal strain of Rhodococcus rhodochrous was resistant to an aromatic fraction of the crude oil (AF), whereas its rough derivatives were sensitive; and so they suggested an association between mucoidal morphology and the ability to tolerate the $\mathrm{AF}^{7}$. Since Rhodococcus sp. 33 forms mucoidal colonies on nutrient agar, we examined a relationship between the mucoidal morphology and the ability to tolerate benzene.

In this study, we isolated morphological mutants from colonies of strain 33 and characterized their tolerance to benzene in order to elucidate mechanisms other than the compositional change in the fatty acids in the cell membranes.

\section{MATERIALS AND METHODS}

\section{Bacterial strain and medium}

Spontaneous rough mutants, R0, R1, R2, R3, R4, and

*Corresponding author. Phone: +81-466-84-3705. Fax: +81-466-84-3354. E-mail: nakajima@brs.nihon-u.ac.jp 
R5, were isolated from Rhodococcus sp. 33. These strains were cultivated on PAS liquid media with either mannitol, benzene or both as carbon sources, as described previously ${ }^{1)}$. Rhodococcus rhodochrous S-2 cells ${ }^{8)}$ were cultivated on IB agar plates ${ }^{9)}$. Nutrient agar (Oxoid, Basingstoke, Hampshire UK) was also used for growth and maintenance of these strains.

\section{Extraction and purification of rhodococcal EPS}

EPS produced by Rhodococcus sp. 33 (33 EPS) and by Rhodococcus rhodochrous S-2 (S-2 EPS) were removed from the cells by shaking and partially purified by enzymatic treatments, phenol/chloroform treatment and ethanol precipitation as described by Urai et al. ${ }^{10)}$ These partially purified EPS were further purified by DEAE-Toyopearl $650 \mathrm{M}$ column chromatography as described by Urai et $a l .{ }^{11)}$

\section{Benzene tolerance test}

Growth conditions: A loopful of bacterial cells grown on nutrient agar plates was inoculated into $50 \mathrm{ml}$ of PAS medium containing $1 \%$ mannitol in a $250-\mathrm{ml}$ shake flask and incubated with shaking for 3 days. After incubation, $2.5 \mathrm{ml}$ of this culture was inoculated into $50 \mathrm{ml}$ of fresh PAS medium containing $1 \%$ mannitol and incubated for $24 \mathrm{~h}$. Then, $2.5 \mathrm{ml}$ of this culture was transferred into 50 $\mathrm{ml}$ of PAS medium equilibrated with benzene in a $250-\mathrm{ml}$ shake flask, and the purified 33 EPS was added to the media at a final concentration of $0.1 \mathrm{mg} / \mathrm{ml}$. The media were incubated with shaking. PAS medium equilibrated with benzene was prepared as follows: Fifty milliliters of PAS medium was dispensed into a 250-ml shake flask, and an empty test tube $(100 \mathrm{~mm} \times 10 \mathrm{~mm} \phi)$ was placed inside the flask. Benzene was added to the test tube to allow its vapor to diffuse into the medium and the headspace. The flask was plugged with cotton to allow diffusion of air into the system. The flask was preincubated with shaking $(100 \mathrm{rpm})$ for $1 \mathrm{~h}$ before inoculation. All procedures were performed at $28^{\circ} \mathrm{C}$.

Resting conditions: Bacterial cells grown on nutrient agar plates were harvested and resuspended in $37.5 \mathrm{mM}$ potassium-phosphate buffer ( $\mathrm{pH}$ 6.9) at approximately $10^{9}$ $\mathrm{cfu} / \mathrm{ml}$. Two milliliters of the cell suspension were transferred into $50 \mathrm{ml}$ of $37.5 \mathrm{mM}$ potassium-phosphate buffer (pH 6.9) equilibrated with benzene as described above, and the purified 33 EPS was added to the buffer at a final concentration of $0.1 \mathrm{mg} / \mathrm{ml}$. The cell suspensions were incubated with shaking for $1 \mathrm{~h}$. All procedures were performed at $28^{\circ} \mathrm{C}$.

\section{Analysis of neutral sugars and uronic acid}

The purified 33 EPS was hydrolyzed as follows: Ten microliters of 33 EPS aqueous solution $(5 \mathrm{mg} / \mathrm{ml})$ was mixed with $20 \mu \mathrm{l}$ of $99 \%$ trifluoroacetic acid, incubated at $100^{\circ} \mathrm{C}$ for $18 \mathrm{~h}$, and then dried in vacuo. The hydrolyzate was dissolved in $10 \mu \mathrm{l}$ of MilliQ water, mixed with
$40 \mu$ of 4 -aminobenzoic acid ethyl ester (ABEE) labeling reagent (Seikagaku Corporation, Tokyo, Japan), and heated at $80^{\circ} \mathrm{C}$ for $1 \mathrm{~h}$. After cooling to room temperature, 200 $\mu \mathrm{l}$ of MilliQ water and $200 \mu \mathrm{l}$ of chloroform were added; and the 33 EPS was then centrifuged at $10,000 \times g$ for 10 min. The aqueous phase was recovered and filtered through a $0.2-\mu \mathrm{m}$-pore-size membrane filter to remove particulate materials. ABEE-labeled saccharides were analyzed by high-performance liquid chromatography (HPLC; Shimadzu LC-6A; Shimadzu, Kyoto, Japan) with a Honenpak C18 column $(75 \mathrm{~mm} \times 4.6 \mathrm{~mm} \phi$; Honen Co., Tokyo, Japan) and a mixture of $200 \mathrm{mM}$ borate buffer and acetonitrile $(93: 7, \mathrm{~V} / \mathrm{V})$. A mixture of L-arabinose, Lfucose, D-galactose, D-glucose, D-mannose, L-rhamnose, Dxylose, D-galacturonic acid, and D-glucuronic acid (1 $\mathrm{mg} / \mathrm{ml}$ each) was treated as described above and then employed as authentic substances for reference.

\section{Chemical modification of 33 EPS}

Carboxyl reduction of 33 EPS: Ten milligrams of 33 EPS was reduced as described by Richards et al. ${ }^{12)}$ The HPLC analysis of the reduced 33 EPS hydrolyzate revealed that most of the glucuronic acid residues had been reduced to glucose (data not shown).

Partially-hydrolyzed 33 EPS: Ten milligrams of 33 EPS was dissolved in $10 \mathrm{ml}$ of $2 \%$ trifluoroacetic acid, heated at $100^{\circ} \mathrm{C}$ for $3 \mathrm{~h}$, and then lyophilized. The lyophilized sample was dissolved in $1 \mathrm{ml}$ of $0.2 \mathrm{M}$ acetic acid, applied onto a Bio-Gel P-2 gel filtration column $(900 \mathrm{~mm} \times 15$ $\mathrm{mm} \phi$ ), and eluted with $0.2 \mathrm{M}$ acetic acid. Fractions containing oligosaccharides ranging from pentamers to dodecamers were combined and lyophilized (data not shown). Almost all of the acetal bonds between pyruvic acid and saccharide chain should be cleaved under the conditions of hydrolyzation described above.

\section{Analytical methods}

Reducing sugar content was determined by the phenolsulfuric acid method ${ }^{13)}$. Pyruvic acid content was enzymatically determined with an F-kit Pyruvic acid (J. K. International Co., Tokyo, Japan), as described by the supplier, after hydrolysis with $2 \%$ TFA at $100^{\circ} \mathrm{C}$ for $120 \mathrm{~min}$. Fatty acid analyses were performed as described previously ${ }^{10)}$.

Gel filtration column chromatography for native 33 EPS was performed by using Sephacryl S1000 $(850 \mathrm{~mm} \times 15$ mm $\phi$; Amersham Biosciences UK Limited, Buckinghamshire, UK) as resin, $1 \mathrm{mM}$ Tris- $\mathrm{HCl}$ (pH8.0) containing $0.1 \mathrm{M} \mathrm{NaCl}$ as eluent, and Dextran T2000 (Amersham Biosciences UK Limited, Buckinghamshire, UK) as size marker.

The purified 33 EPS was dissolved in $0.15 \mathrm{M}$ Tris- $\mathrm{HCl}$ (pH 7.4) at $1 \mathrm{mg} / \mathrm{ml}$, and its reduced viscosity was determined by using an Ostwald viscosity meter No. 1 operated at $27^{\circ} \mathrm{C}$. 


\section{RESULTS}

\section{Isolation of rough mutant strains}

Six spontaneous rough mutants, R0, R1, R2, R3, R4, and R5, were isolated from Rhodococcus sp. 33 on nutrient agar plates or PAS agar plates containing $1 \%$ mannitol as the carbon source. In the presence of benzene these spontaneous rough mutants showed no or little growth $\left(\mathrm{OD}_{600}<0.1\right)$, whereas their parental strain, Rhodococcus sp. 33, showed good growth $\left(\mathrm{OD}_{600}=0.65 \pm 0.04\right)^{1)}$, which was as good as the growth on mannitol as carbon source (Table 1). These rough strains did not produce the extracellular polysaccharides (EPS), whereas the parental strain did.

\section{Purification and physicochemical characterization of 33 EPS}

EPS produced by the Rhodococcus sp. 33 was extracted and purified by ion-exchange chromatography (Fig. 1). The purified 33 EPS was eluted from the Sephacryl S1000 gel-filtration column as a broad single peak earlier than Dextran T2000 (Fig. 2), suggesting its apparent molecular weight to be greater than 2,000,000.

The purified 33 EPS was a white fibrous material soluble in water and alkalis but not in acids, methanol, ethanol, acetone, or dimethyl sulfoxide. Spectrophotometrically, absorption was detected at neither $280 \mathrm{~nm}$ nor $255 \mathrm{~nm}$, suggesting that the 33 EPS preparation did not contain proteins or nucleic acids. Its aqueous solution was viscous, and its reduced viscosity was determined to be $15.6 \mathrm{dl} \mathrm{g}^{-1}\left(=1.56 \mathrm{~m}^{3} \mathrm{~kg}^{-1}\right)$ by the use of an Ostwald viscosity meter.

The lyophilized 33 EPS was hydrolyzed by TFA, labeled with ABEE, and analyzed by HPLC. Consequently, D-galactose, D-glucose, D-mannose, and D-glucuronic acid were found (Fig. 3). Based on these data, the proportions of the constituent sugars $(\% \mathrm{~W} / \mathrm{W})$ were calculated to be the following: D-galactose, $22.1 \%$; D-glu- cose, 22.4\%; D-mannose, 21.5\%; and D-glucuronic acid, $18.4 \%$.

Pyruvic acid was detected in the hydrolysate of 33 EPS obtained with $2 \%$ TFA, and its content was calculated to be $15.6 \%$ (W/W). Fatty acids other than pyruvic acid were not detected in either acid- or alkali-hydrolyzed 33 EPS.

\section{Benzene tolerance test}

Rough mutant strains showed no or little growth in the presence of benzene, whereas their parental strain Rhodococcus sp. 33 showed good growth in either the presence or absence of benzene (Table 1). By the addition of the purified 33 EPS the rough strains showed good growth $\left(\mathrm{OD}_{600}>0.5\right)$ even in the presence of benzene. Without the addition of carbon sources all of the strains showed no or little growth $\left(\mathrm{OD}_{600}<0.1\right)$ on PAS medium containing the purified 33 EPS at $0.1 \mathrm{mg} / \mathrm{ml}$. These data suggest that the rough mutants have no or few defects in their benzene-assimilation system.

Next, we examined the effects of EPS on the toxicity of benzene toward the rough strain, R0, under the resting conditions described in the Materials and Methods (Table 2). After exposure to benzene for $1 \mathrm{~h}, \mathrm{R} 0$ showed great sensitivity to benzene under the resting conditions $(0.19 \%$ survival rate). In the presence of 33 EPS at a final concentration of $0.1 \mathrm{mg} / \mathrm{ml}$, the survival rate of R0 increased to $91 \%$ after exposure to benzene for $1 \mathrm{~h}$, suggesting the protective effect of 33 EPS against benzene toxicity. Although after 1-h incubation in the presence of benzene the cell numbers of strain 33 were slightly higher than in its absence, the cells showed no or little growth even in the presence of benzene after longer period of incubation time.

\section{Effects of chemically modified 33 EPS on the benzene tolerance}

Reduced or partially-hydrolyzed 33 EPS was examined for its protective effect against benzene toxicity under the

Table 1. Effects of 33 EPS on the growth of Rhodococcus sp. 33 and its rough mutants in the presence of benzene ${ }^{a}$

\begin{tabular}{|c|c|c|c|c|}
\hline \multirow[b]{2}{*}{ Strain } & \multirow[b]{2}{*}{$\mathrm{EPS}^{\mathrm{b}}$} & \multicolumn{3}{|c|}{ growth $^{\mathrm{c}}$} \\
\hline & & mannitol $^{\mathrm{d}}$ & mannitol + benzene ${ }^{\mathrm{d}}$ & benzene $^{\mathrm{d}}$ \\
\hline Strain 33 & $(-)$ & +++ & +++ & +++ \\
\hline \multirow{5}{*}{$\begin{array}{l}\text { Rough } \\
\text { mutants }\end{array}$} & $(-)$ & +++ & - & - \\
\hline & native 33 EPS & +++ & +++ & +++ \\
\hline & reduced 33 EPS & +++ & - & - \\
\hline & partially-hydrolyzed 33 EPS & +++ & - & - \\
\hline & S-2 EPS & +++ & +++ & +++ \\
\hline
\end{tabular}

${ }^{a}$ Benzene tolerance test under growth conditions was performed as described in the Materials and Methods section.

${ }^{\mathrm{b}}$ EPS concentration used in this test was $0.1 \mathrm{mg} / \mathrm{ml}$. The symbol $(-)$ means no addition.

c The symbols +++ and - indicate good growth $\left(\mathrm{OD}_{600}>0.5\right)$ and no growth $\left(\mathrm{OD}_{600}<0.1\right)$, respectively.

${ }^{\mathrm{d}}$ Mannitol was added to PAS medium at $1 \%$ as its final concentration, and benzene was allowed to diffuse into media as a vapor into the medium. The cultures, in $250-\mathrm{ml}$ shake flasks, were incubated with shaking for 3 days at $28^{\circ} \mathrm{C}$. 
Table 2. Effects of 33 EPS on the survival of resting cells of Rhodococcus sp. 33 and its rough mutant treated with benzene ${ }^{a}$.

\begin{tabular}{lccc}
\hline \multirow{2}{*}{ Strain } & 33 EPS $^{\mathrm{b}}$ & \multicolumn{2}{c}{ Benzene } \\
\cline { 2 - 4 } Strain 33 & $(-)$ & $65(2)$ & + \\
\cline { 2 - 4 } & $(+)$ & $73(3)$ & $110(0)$ \\
\hline \multirow{2}{*}{ Rough mutant R0 } & $(-)$ & $38(1)$ & $0.19(0.03)$ \\
\cline { 2 - 4 } & $(+)$ & $86(5)$ & $91(11)$ \\
\hline
\end{tabular}

aThe 33 EPS concentration used in this test was $1 \mathrm{mg} / \mathrm{ml}$. The values are percentages of the initial viable cell number $((3.5 \pm$ $0.1) \times 10^{7}$ ). The values in parentheses are standard deviations.

${ }^{\mathrm{b}}$ EPS concentration used in this test was $0.1 \mathrm{mg} / \mathrm{ml}$. The symbol ( - ) means no addition.

growth conditions described in Materials and Methods (Table 1). After the addition of either reduced or partially-hydrolyzed 33 EPS the rough strains showed no growth in the presence of benzene. Finally, we examined the effect of S-2 EPS on the benzene toxicity under growth conditions and found this polysaccharide to protect the rough strains against the benzene toxicity.

\section{DISCUSSION}

In this study we showed that the rough strains derived from Rhodococcus sp. 33 were more sensitive to benzene than the parental mucoidal strain under either the growth or the resting conditions that contained up to 200 p.p.m benzene ${ }^{1)}$. These rough strains did not produce EPS, and the addition of 33 EPS improved their growth and survival in the presence of benzene (Tables 1 and 2). These data suggest that 33 EPS plays an important role in benzene tolerance of Rhodococcus sp. 33, especially in its ability to survive an initial challenge with benzene.

Even in the presence of purified 33 EPS the rough strains showed no or little growth on PAS medium saturated with benzene $(1.8 \mathrm{~g} / \mathrm{l})$ by the addition of liquid benzene (data not shown), whereas Rhodococcus sp. 33 grew well under such conditions ${ }^{1}$. The data suggest that the protective effects to benzene of endogenous 33 EPS might be higher than those of the added 33 EPS.

The mechanism of tolerance to benzene associated with 33 EPS is unknown. Since rough strains of Rhodococcus have strongly hydrophobic surfaces, the direct contact between such cells and benzene may enable the microorganisms to take up the hydrocarbon rapidly. It may be that in the presence of 33 EPS the influx of benzene into the cells might be decreased due to either covering of benzene with 33 EPS micelles and/or a change in cell-surface hydrophobicity.

There are several reports demonstrating that the level of tolerance to hydrocarbons is elevated by the production of various surface-active compounds ${ }^{14,15}$. Emulsification was observed when either 33 EPS or S-2 EPS was mixed with various oils. Therefore, one of the possible mechanisms

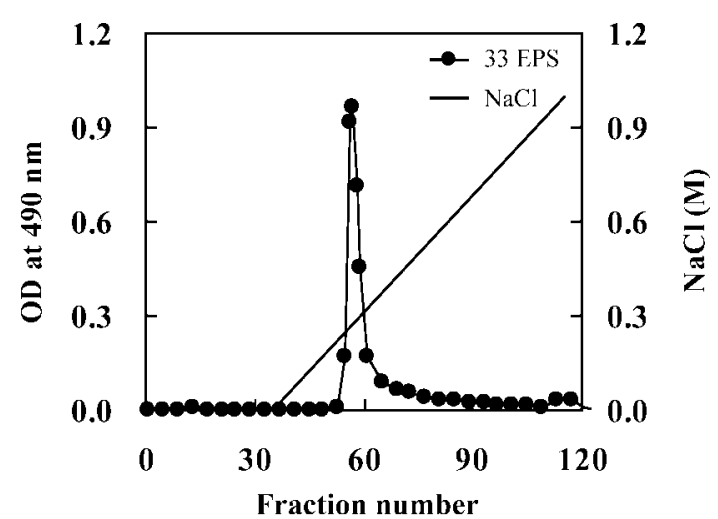

Fig. 1. Ion-exchange chromatography of 33 EPS. Forty milligrams of 33 EPS were dissolved in $40 \mathrm{ml}$ of $10 \mathrm{mM}$ Tris- $\mathrm{HCl}$ buffer, applied to a DEAE-Toyopearl column $(180 \mathrm{~mm} \times 25$ $\mathrm{mm} \phi)$, and eluted with a $600-\mathrm{ml}$ linear gradient $(0-1 \mathrm{M})$ of $\mathrm{NaCl}$.

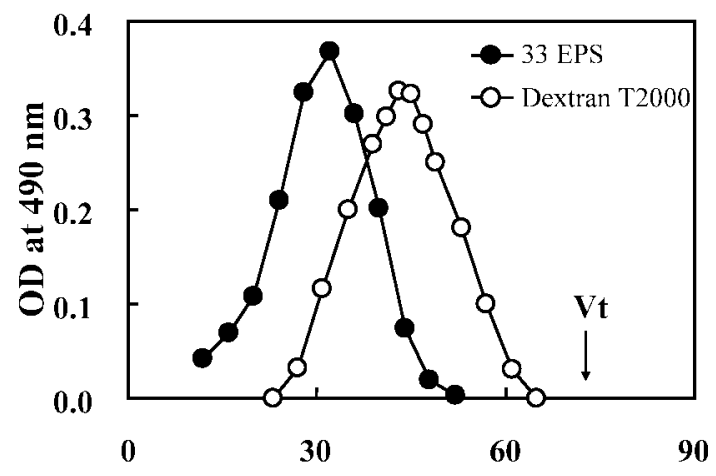

Fraction number

Fig 2. Gel filtration column chromatography of 33 EPS. One milligram of 33 EPS (closed circles) was applied to a Sephacryl S1000 gel filtration column $(850 \mathrm{~mm} \times 15 \mathrm{~mm} \phi)$, and Dextran T2000 (open circles) was employed as a size marker. The arrow indicates the total volume of the gel $(\mathrm{Vt})$.

A

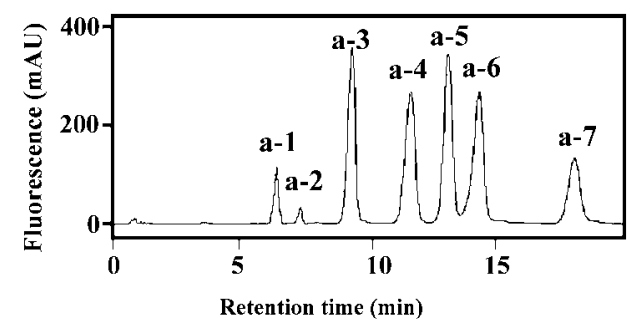

B

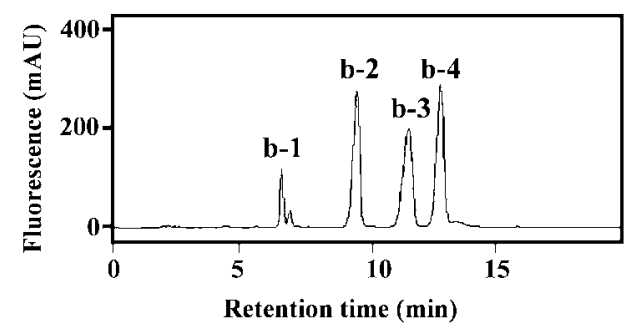

Fig. 3. HPLC patterns of neutral sugars and uronic acid from 33 EPS. Panel A, standard samples; and panel B, 33 EPS. Standard samples: a-1, D-glucuronic acid; a-2, D-galacturonic acid; a-3, D-galactose; a-4, D-mannose; a-5, D-glucose; a-6, L-arabinose; and a-7, D-xylose. 
for tolerance is that the surfactant activity of these EPS molecules renders cells resistant to benzene. Some Rhodococcus strains produce biosurfactants, and these molecules are predominantly glycolipids ${ }^{16)}$. 33 EPS and S-2 EPS are high-molecular-weight acidic polysaccharides and, therefore, are different from previously reported Rhodococcus biosurfactants.

Sunairi et al. reported earlier that S-2 EPS lowered the cell-surface hydrophobicity of rough strains of Rhodococcus, indicating that S-2 EPS functions as a hydrophilin ${ }^{9}$. Aono and Kobayashi reported that a low cell-surface hydrophobicity serves as a defense mechanism against organic solvents ${ }^{17}$. It is thus possible that 33 EPS lowers the cell-surface hydrophobicity and establishes tolerance to benzene in the same way as S-2 EPS.

Rough strains showed good growth in the presence of benzene by the addition of native 33 EPS but not by that of the reduced or the partially-hydrolyzed 33 EPS (Table 1). The S-2 EPS also conferred the benzene tolerance to rough strains under the growth conditions (Table 1), which was reported to be a high-molecular-weight acidic polysaccharide consisting of D-glucose, D-galactose, D-mannose, D-glucuronic acid, stearic acid, and palmitic acid ${ }^{7)}$. These data suggest that acidic residues and the high molecular weight of these rhodococcal EPSs may contribute to the protective effects of these EPSs against benzene toxicity.

Chemical analysis indicated that 33 EPS is an acidic polysaccharide containing 3 kinds of neutral sugars $(66.0 \%$ $\mathrm{W} / \mathrm{W})$, uronic acid (18.4\% W/W), and pyruvic acid (15.6\% $\mathrm{W} / \mathrm{W}$ ), yielding a sum of their contents of $100 \%$. The molar ratio of neutral sugars and uronic acid was estimated to be the following: D-galactose: D-glucose: D-mannose: Dglucuronic acid: pyruvic acid $=1: 1: 1: 1: 1$. Richards' research group reported the chemical structures of EPS produced by 5 different serotypes of the equine pathogen $R$. equi ${ }^{18-22)}$, and the presence of pyruvic acid in its acetal form in EPS of serotypes 1, 3, 4, and 7. Although the molar ratio of neutral sugars, uronic acid, and pyruvic acid of serotype 3 EPS are the same as those of 33 EPS, lactate-substituted hexose was not detected in 33 EPS; whereas serotype 3 EPS was reported to contain lactate-substituted mannopyranose ${ }^{20)}$.

The reduced viscosity of the dilute aqueous solution of the EPS was $15.6 \mathrm{dl} / \mathrm{g},\left(1.56 \mathrm{~m}^{3} \mathrm{~kg}^{-1}\right)$ representing a higher viscosity than that of widely used thickeners, such as carboxymethyl cellulose $\left(3.9 \mathrm{dl} \mathrm{g}^{-1}\left(=3.9 \times 10^{-1} \mathrm{~m}^{3} \mathrm{~kg}^{-1}\right)\right)$ or hydroxyethyl cellulose $\left(8.0 \mathrm{dl} \mathrm{g}^{-1}\left(=8.0 \times 10^{-1} \mathrm{~m}^{3} \mathrm{~kg}^{-1}\right)\right.$, as measured under the same experimental conditions ${ }^{11)}$, thus suggesting the possibility of its application as a thickener.

Thus 33 EPS plays an important role in benzene tolerance of Rhodococcus sp. 33, especially helping the cells to survive an initial challenge with benzene, and its chemical composition affords some insight into the relationship between its chemical structure and function. We are cur- rently studying its chemical structure in terms of the structure/function relationships.

\section{ACKNOWLEDGEMENTS}

We acknowledge members of our laboratories for their technical assistance. This study was supported in part by grants from Sapporo Bioscience Foundation, the program Grants-in-aid for Scientific Research (15580301) of the Japan Society for the Promotion of Science, "Open Research Center Projects" of the Ministry of Education, Culture, Sports, Science and Technology, and Nihon University Multidisciplinary Global Research Grant for 2003. TA and MU were supported by funding from the "Center of Excellence in 21 Century Projects" of the Ministry of Education, Culture, Sports, Science and Technology.

\section{REFERENCES}

1) Paje, M. L.; B. A. Neilan \& I. Couperwhite: A Rhodococcus species that thrives on medium saturated with liquid benzene. Microbiology 143: 2975-2981, 1997

2) Gutierrez, J. A.; P. Nichols \& I. Couperwhite: Changes in whole cell-derived fatty acids induced by benzene and occurrence of the unusual 16:1[omega] 6c in Rhodococcus sp. 33. FEMS Microbiol. Lett. 176: 213-218, 1999

3) Sikkema, J.; J. A. de Bont \& B. Poolman: Mechanisms of membrane toxicity of hydrocarbons. Microbiol. Rev. 59: 201-222, 1995

4) Weber, F. J. \& J. A. de Bont: Adaptation mechanisms of microorganisms to the toxic effects of organic solvents on membranes. Biochim. Biophys. Acta. 1286: 225-245, 1996

5) Heipieper, H. J. \& J. A. de Bont: Adaptation of Pseudomonas putida $\mathrm{S} 12$ to ethanol and toluene at the level of fatty acid composition of membranes. Appl. Environ. Microbiol. 60: 4440-4444, 1994

6) Gutierrez, T.; R. P. Learmonth, P. D. Nichols \& I. Couperwhite: Comparative benzene-induced fatty acid changes in a Rhodococcus species and its benzene-sensitive mutant: possible role of myristic and oleic acids in tolerance. J. Chem. Ecol. 29: 2369-2378, 2003

7) Iwabuchi, N.; M. Sunairi, H. Anzai, M. Nakajima \& S. Harayama: Relationships between colony morphotypes and oil tolerance in Rhodococcus rhodochrous. Appl. Environ. Microbiol. 66: 5073-5077, 2000

8) Wakayama, Y.; M. Nakajima \& H. Murooka: Isolation and characterization of S and R strains of Nocardia sp. CF222. Bull. Coll. Agr. Vet. Med. Nihon Univ. 37: 99-105, 1980

9) Sunairi, M.; N. Iwabuchi, Y. Yoshizawa, H. Murooka, H. Morisaki \& M. Nakajima: Cell-surface hydrophobicity and scum formation of Rhodococcus rhodochrous strains with different colonial morphologies. J. Appl. Microbiol. 82: 204-210, 1997

10) Urai, M.; H. Anzai, N. Iwabuchi, M. Sunairi \& M. Nakajima: A novel moisture-absorbing extracellular polysaccharide from Rhodococcus rhodochrous SM-1. Actinomycetologica 16: 26-31, 2002

11) Urai, M.; H. Anzai, N. Iwabuchi, M. Sunairi \& M. 
Nakajima: A novel viscous extracellular polysaccharide containing fatty acids from Rhodococcus rhodochrous ATCC 53968. Actinomycetologica 18: 15-17, 2004

12) Richards, J. C.; M. B. Perry \& P. J. Kniskern: Structural analysis of the specific polysaccharide of Streptococcus pneumoniae type 9L (American type 49). Can. J. Biochem. Cell. Biol. 62: 1309-1320, 1984

13) Dubois, M.; K. A. Gilles, J. K. Hamilton, P. A. Rebers \& F. Smith: Colorimetric method for determination of sugars and related substances. Anal. Chem. 28: 350-356, 1956

14) Prince, R. C.: Petroleum spill bioremediation in marine environments. Crit. Rev. Microbiol. 19: 217-242, 1993

15) Kurane, R. \& H. Matsuyama: Production of a bioflocculant by mixed culture. Biosci. Biotechnol. Biochem. 58: 15891594, 1994

16) Lang, S. \& J. C. Philp: Surface-active lipids in rhodococci. Antonie Van Leeuwenhoek 74: 59-70, 1998

17) Aono, R. \& H. Kobayashi: Cell surface properties of organic solvent-tolerant mutants of Escherichia coli K-12. Appl.
Environ. Microbiol. 63: 3637-3642, 1997

18) Severn, W. B. \& J. C. Richards: The structure of the specific capsular polysaccharide of Rhodococcus equi serotype 4. Carbohydr. Res. 320: 209-222, 1999

19) Severn, W. B. \& J. C. Richards: Structural analysis of the specific capsular polysaccharide of Rhodococcus equi serotype 2. Carbohydr. Res. 206: 311-332, 1990

20) Severn, W. B. \& J. C. Richards: The acidic specific capsular polysaccharide of Rhodococcus equi serotype 3. Structural elucidation and stereochemical analysis of the lactate ether and pyruvate acetal substituents. Can. J. Chem. 70: 2664-2676, 1992

21) Masoud, H. \& J. C. Richards: Structural elucidation of the specific capsular polysaccharide of Rhodococcus equi serotype 7. Carbohydr. Res. 252: 223-233, 1994

22) Leitch, R. A. \& J. C. Richards: Structural analysis of the specific capsular polysaccharide of Rhodococcus equi serotype 1. Biochem. Cell. Biol. 68: 778-789, 1990 